\title{
CORRIGENDUM
}

\section{Axisymmetric global gravitational equilibrium for magnetized, rotating hot plasma - Corrigendum}

\author{
Peter J. Catto, István Pusztai and Sergei I. Krasheninnikov \\ doi:10.1017/S0022377815001245, Published by Cambridge University Press, \\ December 1, 2015
}

The authors wish to thank Antoine Cerfon and Dimitrios Andriopoulos of the Courant Institute at New York University for pointing out an error in our manuscript (Catto, Pusztai \& Krasheninnikov 2015). This mistake only affects the results when a toroidal magnetic field is present. It arises because our Grad-Shafranov equation (3.11) should be corrected to read

$$
\begin{aligned}
\frac{\mathrm{d}^{2} H}{\mathrm{~d} \mu^{2}}+\frac{\alpha(\alpha+1)}{1-\mu^{2}} H= & \alpha\left\{\beta\left[\frac{g}{2} H^{-1 / \alpha}-\omega^{2}\left(1-\mu^{2}\right) H^{2 / \alpha}-(\alpha+2)\right]\right. \\
& \left.-\frac{(\alpha+1) b^{2} \mathrm{e}^{-\chi}}{\left(1-\mu^{2}\right) H^{2 / \alpha}}\right\} H^{1+4 / \alpha} \mathrm{e}^{\chi}
\end{aligned}
$$

where the only change is to insert the missing $\mathrm{e}^{-\chi}$ multiplying the toroidal magnetic field term proportional to $b^{2}$ since it cannot have any density dependence. There is also a typographical error in (3.6) since there, $B_{o}$ should be replaced by $B_{P o}$, with $B_{P o}=-\alpha \psi_{o} / R_{o}^{2}$. The equations shown here and the material in quotes are the corrected content. The references remain the same as in the publication.

The preceding change in the Grad-Shafranov equation (3.11) changes (3.17) and the short sentence that follows to

$$
\alpha+2=\frac{C^{2} b^{2}-\beta \omega^{2} \mathrm{e}^{-g-\omega^{2}\left(1-C^{-1}\right)}}{C^{3}+C^{2} b^{2}+C \beta \mathrm{e}^{-g-\omega^{2}\left(1-C^{-1}\right)}} .
$$

'Consequently, we expect $\alpha+2<0$ unless $C^{2} b^{2}>\beta \omega^{2} \mathrm{e}^{-g-\omega^{2}\left(1-C^{-1}\right)}$, with $C=1$ if $g=0$ '.

In $\$ 4$ the only change occurs in the penultimate sentence that becomes the following 'For example, we do not consider the limit $C^{2} b^{2} \mathrm{e}^{g+\omega^{2}\left(1-C^{-1}\right)}>\beta \omega^{2} \sim$ $\beta g / 2 \gg \beta \gg 1$, which requires a toroidal magnetic field but allows the magnetic field to vanish at infinity'.

There are a number of changes in $\S 5$ where we keep the toroidal field. Equation (5.1) through to the end of the paragraph including (5.3) should be corrected to read:

$\dagger$ Email address for correspondence: catto@psfc.mit.edu 


$$
\begin{aligned}
& (\alpha-1)(\alpha+2) \int_{0}^{1} \mathrm{~d} \mu H+\alpha(\alpha+1) b^{2} \int_{0}^{1} \mathrm{~d} \mu H^{1+2 / \alpha} \\
& =\alpha \beta \int_{0}^{1} \mathrm{~d} \mu\left(1-\mu^{2}\right) H^{1+4 / \alpha}\left[\frac{g}{2} H^{-1 / \alpha}-\omega^{2}\left(1-\mu^{2}\right) H^{2 / \alpha}-(\alpha+2)\right] \mathrm{e}^{\chi} .
\end{aligned}
$$

'Using the vacuum magnetic field solution for the $\alpha=-2$ root of $H=1-\mu^{2}$, we find that the $b^{2} \sim \beta g \sim \beta \omega^{2} \ll 1 \sim \beta$ corrections to that result must satisfy'

$$
\frac{2(\alpha-1)(\alpha+2)}{3}+\alpha(\alpha+1) b^{2}=\alpha \beta \int_{0}^{1} \mathrm{~d} \mu\left[\frac{g}{2} \sqrt{1-\mu^{2}}-\omega^{2}-(\alpha+2)\right] \mathrm{e}^{-g\left[1-\sqrt{1-\mu^{2}}\right]} .
$$

'For $g \ll 1$ only a weak density departure from cylindrical symmetry is allowed, giving'

$$
\alpha \simeq-2+\left[b^{2}+\beta(\pi g / 8)-\beta \omega^{2}\right] /(1+\beta) .
$$

'Based on (3.17) we expect $b^{2}>\beta \omega^{2}$ is required for a solution that keeps $0>\alpha>-2$, thereby making the poloidal magnetic field fall off at large distances and pinch in slightly at the equatorial plane. Indeed, in this small $g$ limit, finite $b^{2}>\beta \omega^{2}$ seems to be required to find a numerical solution for $\alpha>-2$ '.

Next, equation (5.4) and the remainder of the paragraph that it appears in should be corrected to read as follows

$$
\alpha \simeq-2+b^{2}+\beta\left(g-2 \omega^{2}\right)(\pi / 8 g)^{1 / 2} .
$$

'These results are the same as in Catto \& Krasheninnikov (2015) except the toroidal field term has been retained and it further enhances the pinching in of the flux surfaces at the equatorial plane. The disk thickness from $\mathrm{e}^{-g \mu^{2} / 2}$ is as given by (4.10). Result (5.4) is verified by a numerical solution which is imperceptibly different from that shown in figure $6(a, b)$ for $\beta=0.001, g=100, \omega^{2}=40$ and $b^{2}=0.05$. Analytically we find $\alpha=-1.949$ and $\Delta / R=0.14$ and a sensitive numerical solution is found for $\alpha=$ -1.951214 and $\Delta / R=0.12$. We only need $b^{2}>\beta \omega^{2}$ in this limit to satisfy $\alpha+2>0$ from (3.17) due to the exponential $g$ factor and the use of the vacuum solution for $H$ away from the equatorial plane. Equation (5.4) remains valid in the strict Keplerian case $g=2 \omega^{2} \gg 1$, where we can evaluate the integrals in (5.2) a little more carefully to find $\alpha+2 \simeq b^{2} /\left[1+\beta(\pi / 2 g)^{1 / 2}\right]$, which is consistent with (5.4) when $\beta \ll g^{1 / 2}$. The numerical solution confirms that this strict Keplerian case is a valid limit'.

'Catto \& Krasheninnikov (2015) also find a disk solution localized to the equatorial plane by considering $g>2 \omega^{2}$ and then allowing $g-2 \omega^{2} \gg 1 \gg \beta$, so that the exponential dependence $\mathrm{e}^{x}$ in the Grad-Shafranov equation provides the desired localization about $\mu=0$ for the assumed small $\beta$ terms. Therefore, we modify their treatment to find disk solutions with strong poloidal variation, but with the toroidal magnetic field retained to satisfy (3.17). This constraint was not considered in Catto \& Krasheninnikov (2015). To begin, we need to find a solution in the disk different from the cylindrical solution $H=\left(1-\mu^{2}\right)^{-\alpha / 2}$ valid outside the disk. We find this inner disk solution by considering the approximate Grad-Shafranov equation'

$$
\frac{\mathrm{d}^{2} H}{\mathrm{~d} \mu^{2}}+\alpha(\alpha+1) b^{2} \mathrm{e}^{-(\alpha+2)(1-H) / \alpha} \simeq \alpha \beta\left[\frac{g}{2}-\omega^{2}-(\alpha+2)\right] \mathrm{e}^{\left(g-2 \omega^{2}\right)(1-H) / \alpha},
$$

'where now both rotation and gravity enter the exponential density dependence, for which we use'

$$
H^{1 / \alpha}=\mathrm{e}^{(1 / \alpha) \ln H} \simeq \mathrm{e}^{(1 / \alpha)(H-1)} \simeq 1+(H-1) / \alpha+\cdots .
$$


'When $g-2 \omega^{2} \gg 1 \sim \alpha+2>0$ we obtain strong exponential decay away from the equatorial plane. Very near the equatorial plane $\mathrm{d}^{2} H / \mathrm{d} \mu^{2}<0$ in (5.5) if $g / 2>\omega^{2}+$ $\alpha+2>0$ with $\alpha<0$, but once the right-hand side of (5.5) decays away then the $b^{2}$ term can grow. For $\alpha+2 \sim 1$ and $b^{2}$ not too large this growth occurs far enough away from the equatorial plane that the $b^{2}$ term may be ignored in the disk. These observations suggest, in agreement with Catto \& Krasheninnikov (2015), that solutions that are strongly localized to the equatorial plane in the presence of gravity are not possible for $g<2 \omega^{2}$ and $0>\alpha>-2$ since the rotation is too strong for the plasma to be gravitationally confined'.

'Continuing as in Catto \& Krasheninnikov (2015), we multiply (5.5) (with the $b^{2}$ term ignored) by $\mathrm{d} H / \mathrm{d} \mu$ and integrate from $H=1$ (at $\mu=0$ ) to $H<1$ (for $\mu^{2}>0$ ) to find for $\alpha+2 \sim 1$

$$
\frac{\mathrm{d} H}{\mathrm{~d} \mu} \simeq \alpha \sqrt{\beta\left[1-\mathrm{e}^{\left(g-2 \omega^{2}\right)(1-H) / \alpha}\right]}
$$

'where we select the negative root to make $\mathrm{d} H / \mathrm{d} \mu<0$. Using $\int \mathrm{d} x / \sqrt{1-\mathrm{e}^{-x}}=$ $2 \tanh ^{-1} \sqrt{1-\mathrm{e}^{-x}}$ we obtain'

$$
\frac{g-2 \omega^{2}}{\alpha}(H-1)=x=-\ln \left[1-\tanh ^{2}(\sigma \mu / 2)\right] \rightarrow\left\{\begin{array}{cl}
(\sigma \mu / 2)^{2}+\cdots & \sigma|\mu| / 2 \ll 1 \\
\mp \sigma \mu-\ln 4+\cdots & \sigma|\mu| / 2 \gtrsim 1,
\end{array}\right.
$$

'where $\sigma \equiv\left(g-2 \omega^{2}\right) \sqrt{\beta}$ and the upper (lower) sign is for $\mu>0(\mu<0)$. A solution strongly localized at the equatorial plane is found for $-x=\left(g-2 \omega^{2}\right)(1-H) / \alpha \gg 1$ that results in only a small departure from the gravity free solution $H=\left(1-\mu^{2}\right)^{-\alpha / 2}$ that remains an adequate approximation in the outer region. The behaviour $x \approx \mp \sigma \mu \approx$ $\mp \sigma z / R$ implies a disk width $\Delta=R / \sigma$ so that $\sigma \gg 1$ is required'.

'Using (5.7) and (5.8) on the right-hand side of the integral constraint (5.1), with the cylindrical solution $H=\left(1-\mu^{2}\right)^{-\alpha / 2}$ inserted on the left-hand side, yields the approximate result'

$$
\alpha+2 \simeq \frac{b^{2}}{1+b^{2}}+\sqrt{\beta}-O\left(\beta \omega^{2}\right) \simeq \frac{b^{2}}{1+b^{2}} .
$$

'Gravity is assumed negligible outside the disk in this $\beta \ll 1$ limit, where the solution becomes cylindrical (with $C \simeq 1$ ). Then (5.9) is in agreement with (3.14) provided we assume $1 \sim b^{2} \gg \beta \omega^{2} \sim \sqrt{\beta}$ so the outer solution is well approximated by $H=$ $\left(1-\mu^{2}\right)^{-\alpha / 2}$. The plasma disk width is given by'

$$
\Delta / R=1 /\left[\beta^{1 / 2}\left(g-2 \omega^{2}\right)\right] \ll 1,
$$

'requiring $1 /\left(g-2 \omega^{2}\right)^{2} \ll \beta \ll 1$. Strict Keplerian motion is not allowed in this low $\beta$ thin disk limit'.

'The new figure $7(a, b)$ shows the flux surfaces, density contours and $H$ for $\beta=$ $0.01, g=120, \omega^{2}=10$ and $b^{2}=1$, for which the analytic results give $\Delta / R=0.1$ and $\alpha=-1.5$. The numerical solution gives $\Delta / R=0.0938$ and $\alpha=-1.4715$. In $(b)$ we also plot $H=\left(1-\mu^{2}\right)^{-\alpha / 2}$ for reference. In this case the density decreases with radius (since $\alpha>-1.5$ ). For the same parameters but with $b^{2}=1$ we find $\alpha=-1.33$ and $\Delta / R=0.1$ versus the numerical values of $\alpha=-1.314$ and $\Delta / R=0.094$. We have not found solutions for $\alpha \rightarrow 0$, as claimed in Catto \& Krasheninnikov (2015), even for finite $b^{2}$. 

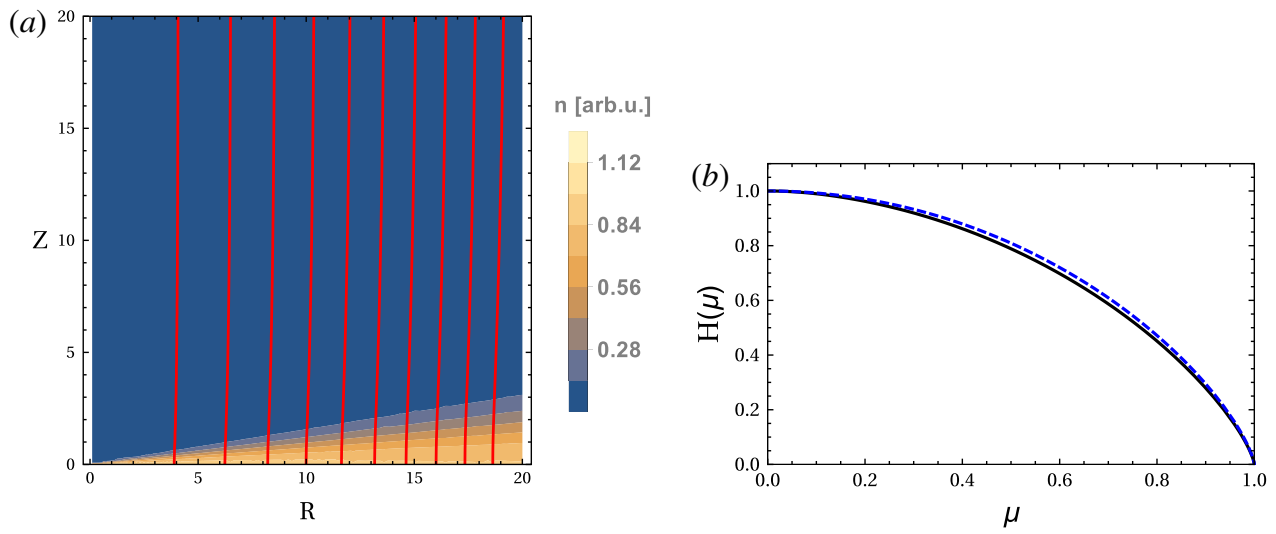

Figure 7. Parameters $g=120, \omega^{2}=10, \beta=0.01$ and $b^{2}=1$, giving $\alpha=-1.4715$. (a) Magnetic surfaces (red curves) and density (colour shading) as functions of $R$ and $Z$. The density is normalized to unity at $R=1$ and $Z=0$. (b) The solution $H(\mu)$ is plotted versus $\mu$. The numerical result is shown as the solid curve and $\left(1-\mu^{2}\right)^{-\alpha / 2}$ is shown dashed for reference.

\section{Acknowledgements}

Work supported by the US Department of Energy grants DE-FG02-91ER-54109 at MIT and DE-FG02-04ER54739 at UCSD and by the International Career Grant of Vetenskapsrådet (Dnr. 330-2014-631).

\section{REFERENCES}

Catto, P. J. \& Krasheninnikov, S. I. 2015 A rotating and magnetized three-dimensional hot plasma equilibrium in a gravitating field. J. Plasma Phys. 81, 105810301.

Catto, P. J., Pusztai, I. \& Krasheninnikov, S. I. 2015 Axisymmetric global gravitational equilibrium for magnetized, rotating hot plasma. J. Plasma Phys. 81, 515810603. 